\title{
Floristic diversity, origins and properties of city trees in Burkina Faso
}

\author{
Souleymane GANABA \\ Environment and Forestry Department, Institute for Environment and Agricultural Research (INERA), National \\ Centre for Scientific and Technological Research (CNRST), BP 7047 Ouagadougou 03, Burkina Faso. \\ E-mail: ganabasouley@gmail.com; Phone: +22670323258
}

\begin{abstract}
In Burkina Faso, insufficient or partial information exists on the forest potential of urban areas, marked mainly by the introduction of many exotic species. The obje0ctive of this study was to assess the floristic diversity of city trees, to reveal the benefits and risks associated with their properties in a context of climate change, increasing demography and accelerated urbanization. Surveys on 49 urban municipalities were conducted from 2015 to 2020 to identify and describe species at different phenological stages to enable photographic image recognition. Confirmations are made with floras, previous works and documentary compilations. The analyses relate to the biological diversity of species, their origins and uses. The floristic diversity of Burkina Faso's city trees consists of 251 species belonging to 174 genera and 58 families. It is comprised of 178 introduced species and 73 native species. The most represented families are Arecaceae with 7.97\% of species, Euphhorbiaceae (7.57\%), Moraceae (6.37\%), Fabaceae - Mimosoideae (5.98\%), Apocynaceae $(5.98 \%)$ and the Fabaceae Caesalpinioideae (5.58\%). The most common species are Khaya senegalensis, Mangifera indica and Azadirachta indica. This work has helped to characterize the city trees of Burkina Faso. It will update the flora of the country, which is enriched with 36 new exotic species.
\end{abstract}

(C) 2020 International Formulae Group. All rights reserved.

Keywords: Urban tree, characterization, biological diversity, communes.

\section{INTRODUCTION}

Vegetation degradation as well as erosion of floristic diversity over the past four decades in most Sahel countries in general and in Burkina Faso in particular, have been documented by several studies (Ganaba, 2011; Kadeba et al., 2014). For the period 1999-2002, the second State of the Environment Report in Burkina Faso (REEB-II) states that 20,968 hectares of forests have been transformed into agricultural territories, or $2.33 \%$ of the forest sector. Approximately 1,444,316 hectares of steppes and savannahs have been partially or completely converted to agricultural land, or $10.66 \%$ of the total area of steppes and savannahs).
Furthermore, insufficient or partial information exists about the forest potential in urban areas (Ouédraogo, 2005; Soma, 2012), marked mainly by introduction of many exotic species. In addition to that, there is limited knowledge about the functions and uses of cities trees. Moreover, the country's rapid population growth and rapid urbanization reflect of the West African sub-region with rates of 5\% in 1900, 12\% in 1950, 28\% in 1980 and $17 \%$ in 2000 (Fuwape and Onyekwelu, 2011). This is why there is a growing awareness in Africa of the need for urban forests and parks to satisfy physical and material needs and for recreation (Çay, 2016). However, information on the nature and level 
of urban forest resources is essential for developing sustainable management strategies. Knowledge of the city's forestry potential requires an assessment of the tree heritage of the municipalities or "communes". A good assessment requires knowledge of the flora. The objective of the study is to evaluate the floristic diversity of city trees, to reveal the benefits and risks associated with their properties in a context of climate change, growing demography and accelerated urbanization.

\section{MATERIALS AND METHODS Study area}

The study area covered as much as possible the chief towns the 49 urban communes of Burkina Faso with Ouagadougou as capital city (Figure 1). Burkina Faso is a landlocked Sahelian country in the Niger loop. It is bordered to the north and west by Mali, to the east by Niger and to the south by Benin, Togo, Ghana and Côte d'Ivoire. The climate is Sudanian and Sahelian, alternating a dry season from October to May and a short rainy season from June to September. Average monthly temperatures vary between $30-34{ }^{\circ} \mathrm{C}$ in March - April and between $23-25^{\circ} \mathrm{C}$ in December January. Sunshine lasts more than 10 hours per day and evaporation exceeds 2,600 $\mathrm{mm}$ per year. Most of Burkina Faso's territory (about $75 \%$ ) rests on an old Precambrian crystalline basement which gives it a generally flat and monotonous relief.

The phytogeographical division in Burkina Faso (Figure 1) distinguishes two domains (Guinko, 1984): the Sahelian phytogeographic domain subdivided into two phytogeographical sectors (strict Sahelian phytogeographic sector and sub-Sahelian phytogeographic sector) and the Sudan phytogeographical domain comprising the northern Sudanese and south Sudan sectors. The population is estimated at 20,870,060 in 2019 on an area of $270,764 \mathrm{~km}^{2}$ and a population growth of $3.05 \%$ per year (Population Dataset, 2020). More than $40 \%$ of the population still lives below the poverty line, according to Prince and Fantom (2014). The country's economy is dominated by agriculture and livestock, which account for more than $85 \%$ of the population.
The urbanization rate, which stood at only $4.8 \%$ of the population in 1960 , has been accelerating since 1985 , from $12.7 \%$ to $22.7 \%$ in 2006. For example, the population of the city of Ouagadougou, which was estimated at 709,736 in 1996 has grown to $1,475,839$ in 2006 and estimated at 3,000,000 in 2019. Its population, which grows by $7.2 \%$ per year, is one of the highest rates in the world and also poses a serious problem in terms of pollution, transport, safety, health and education. The country's second largest city, Bobo Dioulasso, has seen its population grow even faster than the capital, at about $11 \%$ per year, and could exceed one million in a short time (0.8 million in 2017) (Population Dataset, 2020).

In urban centres, climate change and increasing urbanization increase the need for wood (fire, construction, (Ouédraogo, 2006), the risks of pollution and nuisance, but also the degradation of peri-urban vegetation.

\section{Data collection}

The city tree here refers to any woody or similar material present in the city, whether spontaneous or introduced by man. City trees include both concession trees and offconcession trees. Concession trees are all woody plants (tree, shrub and subshrub), present within the boundaries of the residential or service concession. Out-of-concession trees are alignment shafts and trees located in urban forests, urban parks, botanical gardens, green spaces, greenbelts, peri-urban plantations and agroforestry reserves.

Prospecting trips to the cities of Burkina Faso's, 49 urban municipalities were organized from 2015 to 2020. Descriptions and photographic shots of several shots at different phonological phases (leafing, leaf removal, flowering, fruiting, etc.) of each plant were made to allow for further recognition. Verification of the determination was confirmed using flores (Lebrun and Stork (1991, 1992, 1995, 1997), Sacandé et al. (2012), Thiombiano et al. (2012), Arbonnier (2002).

The nomenclature used is that of Lebrun and Stork (1991, 1992, 1995, 1997) and Kyalangalilwa et al. (2013) for acacias. 


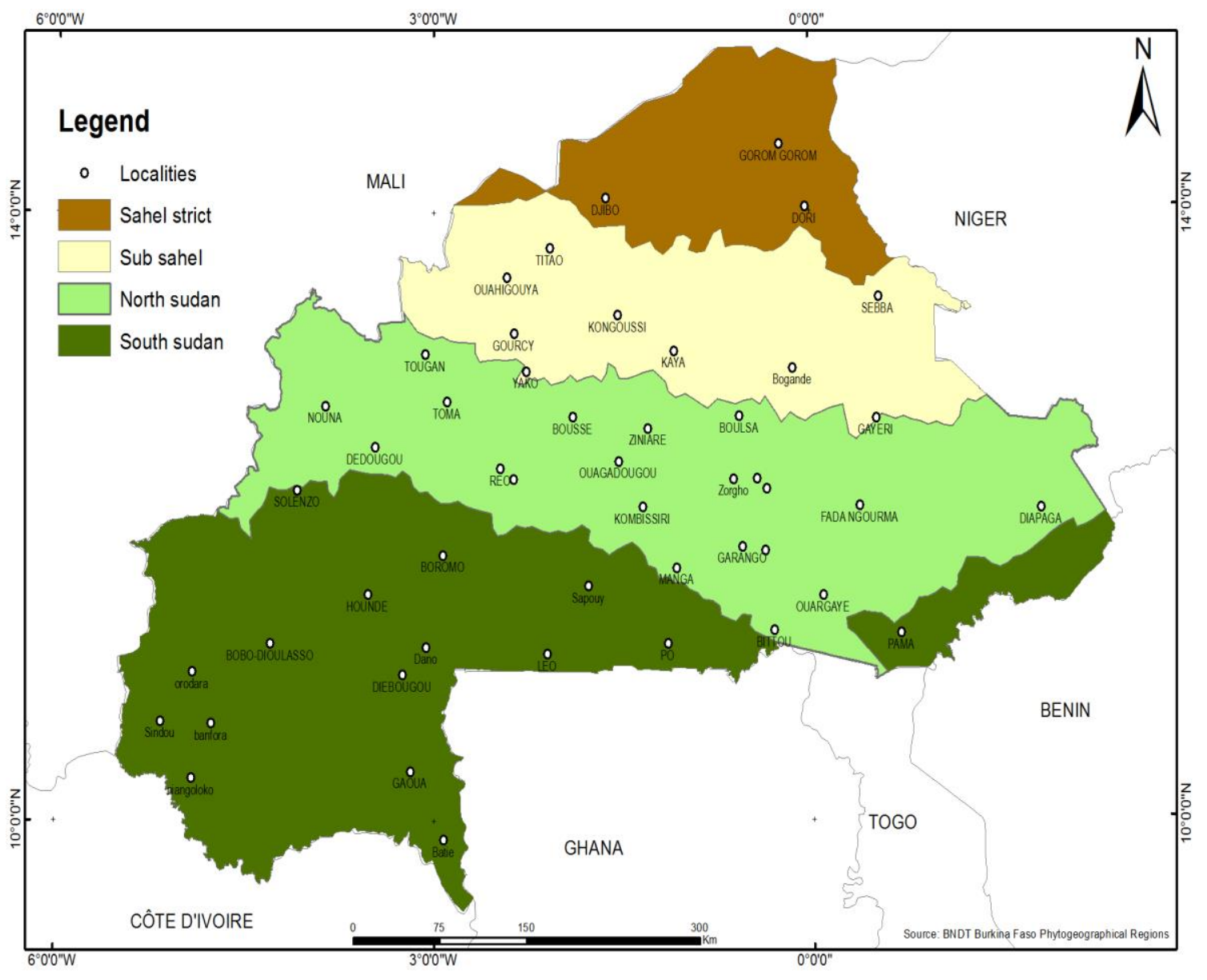

Figure 1: Distribution of Burkina Faso's urban municipalities by phytogeographic zone (IGB, Ouagadougou).

\section{RESULTS \\ Floristic Diversity}

The floristic diversity of Burkina Faso's city trees consists of 251 species belonging to 174 genera in 58 families. It also includes $1.6 \%$ of Gymnosperms (4 families), $5.9 \%$ of Monocotyledons (2 families) and $92.5 \%$ of Dicotyledons (52 families, Table 1). There are 178 introduced species and 73 native ones (Figure 2).

The most represented families are the Arecaceae, which account for $7.9 \%$ of the species, Euphhorbiaceae (7.5\%), Moraceae (6.4\%), Fabaceae - Mimosoideae (5.9\%), Apocynaceae (5.9\%), FabaceaeCaesalpinioideae (5.5\%), Rubiaceae (4.7\%), Malvaceae (3.9\%) Rutaceae (3.6\%), FabaceaeFaboideae (3.6\%), Anacardiaceae (3.2\%) and
(2.4\%) for each of families of Verbenaceae, Bignoniaceae and Acanthaceae (Figure 3).

\section{Provenance of city trees}

The diversity of city trees in Burkina Faso is dominated by African species (native and African origin) which represent $34 \%$ of the species. Then follow the species from Asia that represent $27 \%$ of the species and the species of American origin which represent $24 \%$ of the total. Australian, Caribbean and Malagasy species are represented by $5 \%, 3 \%$ and $4 \%$ respectively. The contribution of Europe and the Mediterranean basin is small and less than $2 \%$ of species (Figure 3 ). The areas of high transfer of phylogenetic resources for city trees in Burkina Faso are: Southeast Asia (11.5\%), South America (10.7\%), Tropical South Asia 
(9.9\%), Australia and Zealand (4.8\%), Madagascar $3.8 \%$ of species and North America (3.8\%). The 73 native species account for $29 \%$ of the total species.

Species from South America include species such as Allamanda blanchetii A. DC., Allamanda cathartica L., Anacardium occidentale L., Annona spp. Trees native to Southeast Asia are represent by Aralia filicifolia Ridl., Araucaria heterophylla (Salisb.) Franco, Artocarpus altilis (Parkinson) Fosberg, etc. Plants native to South Asia are : Argyreia nervosa (Burm. f.) Bojer, Artocarpus heterophyllus Lam., etc. The plants introduced from Australia and Zealand are: Acacia holosericea A. Cunn. ex G. Do, Acalypha wilkesiana Muüll. Arg., Aralia balfouriana André, etc. The plants native to North America are: Washingtonia filifera (Linden ex André) H.Wendl., Washingtonia robusta H.Wendl., Caesalpinia pulcherrima (L.) Sw., etc. The plants of Mediterranean origin are :
Chamaerops humilis L., Jasminum fruticans L., Viburnum tinus L. et Vitis vinifera L. The plants introduced from Madagascar are: Bismarckia nobilis Hildebrandt \& H. Wendl, Delonix regia (Bojer ex Hook.) Raf., Dypsis madagascariensis (Becc.) Beentje et $\mathrm{J}$. Dransf., etc. The plants introduced from West Africa are: Cola nitida (Vent.) Schott \& Endl., Euphorbia basalmifera Aiton, Ficus lutea Vahl, etc. (Table 1).

Native plants of 73 species are distributed in 27 families. The most representative are

Fabaceae-Mimosoideae with 9 species, Malvaceae and Moraceae with 6 species each,

Fabaceae-Caeslpiniaceae

and Apocynceae and Arecaceae with 5 species each and Fabaceae-

Faboideae, Anacarciadeae, Capparaceae with 4 species each. They are recorded in the following Table 2.

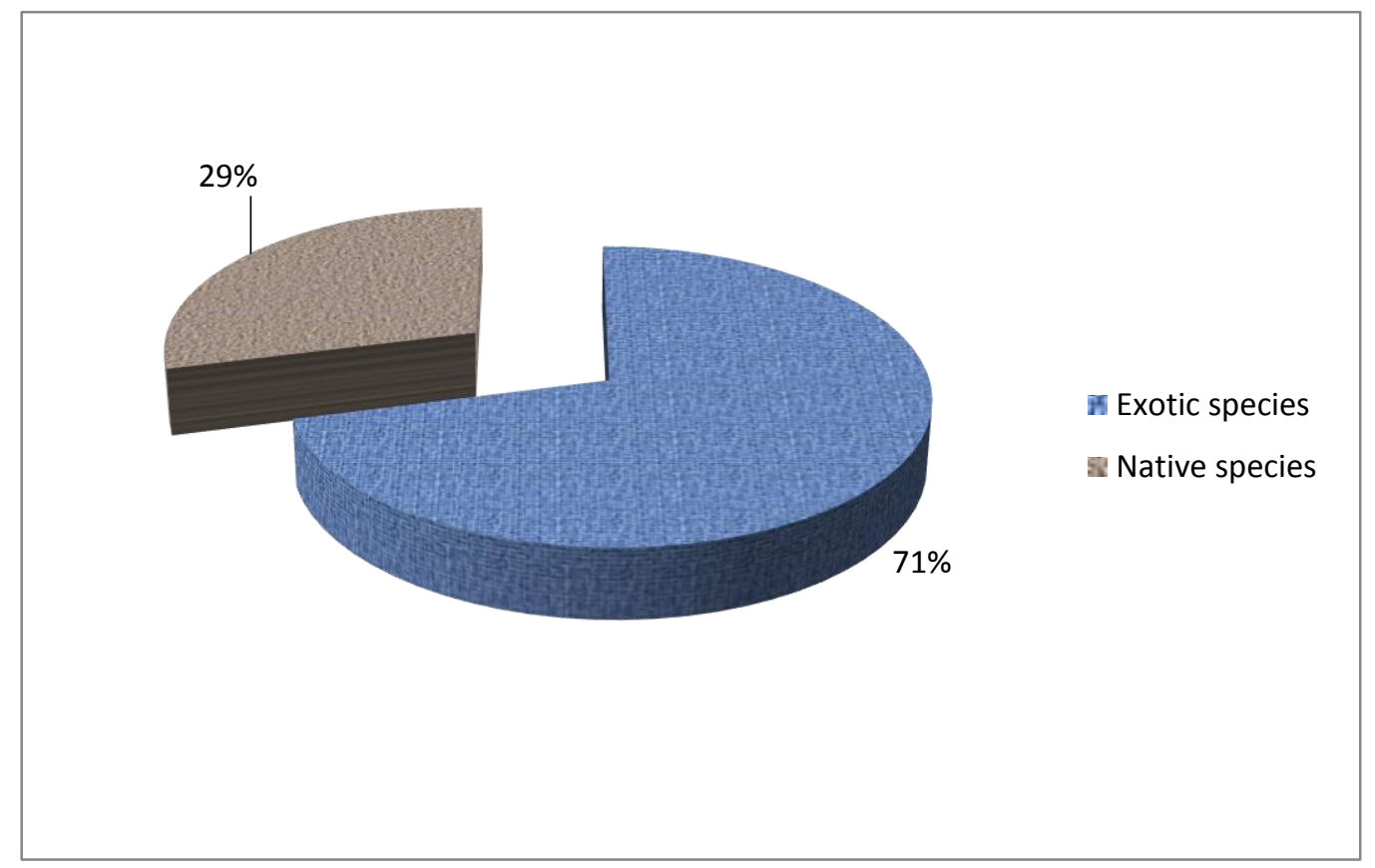

Figure 2: Distribution of Burkina Faso city trees in Exotic and Native Species. 
Table 1: Burkina Faso urban trees species distribution by family and provenance.

\begin{tabular}{|c|c|c|c|}
\hline Class & Family & Species & Provenance \\
\hline & Arecaceae & Bismarckia nobilis Hildebrandt \& H. Wendl & Madagascan \\
\hline & & Borassus aethiopum L. & Native \\
\hline & & Borassus akeasii Bayton, Ouédraogo \& Guinko & Native \\
\hline & & Caryota mitis Lour. & South America \\
\hline & & Chamaedora elegans Mart. & South America \\
\hline & & Chamaerops humilis L. & Mediterranean \\
\hline & & Cocos nucifera $\mathrm{L}$. & South America \\
\hline & & Dypsis madagascariensis (Becc.) Beentje et J. Dransf. & Madagascan \\
\hline & & Elaeis guineensis Jacq. & Western Africa \\
\hline & & Hyophorbe lagenicaulis (L.H.Bailey) H.E.Moore & Madagascan \\
\hline & & Hyphaene thebaica (L.) Mart. & Native \\
\hline & & Phoenix canariensis Hort. ex Chabaud & Western Africa \\
\hline & & Phoenix dactylifera $\mathrm{L}$. & $\begin{array}{l}\text { Northern Africa, } \\
\text { Western Asia }\end{array}$ \\
\hline & & Phoenix sylvestris (L.) Roxb. & Southern Asia \\
\hline & & Raphia sudanica A. Chev. & Native \\
\hline & & $\begin{array}{l}\text { Rhopalostylis sapida (Sol. ex G.Forst.) H.Wendl. \& } \\
\text { Drude }\end{array}$ & $\begin{array}{l}\text { Australia and } \\
\text { Zealand }\end{array}$ \\
\hline & & Roystonea regia (Kunth) O.F.Cook & Central America \\
\hline & & Trachycarpus fortunei (Hook.) H.Wendl. & South-Eastern Asia \\
\hline & & Washingtonia filifera (Linden ex André) H.Wendl. & Northern America \\
\hline & & Washingtonia robusta $\mathrm{H}$. Wendl. & Northern America \\
\hline & & Wodyetia bifurcata A.K. Irvine & $\begin{array}{l}\text { Australia and } \\
\text { Zealand }\end{array}$ \\
\hline & Poaceae & Dendrocalamus giganteus WALL ex. Munr & South-Eastern Asia \\
\hline Monocotylédones & & Oxytenanthera abyssinica (A.Rich.) Munro & Native \\
\hline \multirow{11}{*}{ Dicotyledones } & \multirow[b]{6}{*}{ Acanthaceae } & Aphelandra squarrosa Nees & $\begin{array}{l}\text { South and Northern } \\
\text { America }\end{array}$ \\
\hline & & Barleria cristata $\mathrm{L}$. & Southern Asia \\
\hline & & Strobilanthes dyerianus (Lodd. et al.) T. Anderson & $\begin{array}{l}\text { Madagascan, } \\
\text { Southern Asia }\end{array}$ \\
\hline & & Thunbergia erecta (Benth.) T. Anderson & Tropical Africa \\
\hline & & Thunbergia grandiflora Roxb., 1832 & Tropical Africa \\
\hline & & Sanchezia speciosa Leonard & South America \\
\hline & Adoxaceae & Viburnum tinus L. & Mediterranean \\
\hline & \multirow[t]{4}{*}{ Anacardiaceae } & Anacardium occidentale $\mathrm{L}$. & South America \\
\hline & & Haematostaphis barteri Hook $\mathrm{f}$. & Native \\
\hline & & Lannea microcarpa Engl. \& K. Krause & Native \\
\hline & & Mangifera indica $\mathrm{L}$. & Southern Asia \\
\hline
\end{tabular}




\begin{tabular}{|c|c|c|}
\hline & Sclerocarya birrea (A. Rich.) Hochst. subsp. birrea & Native \\
\hline & $\begin{array}{lcc}\text { Sclerocarya } \quad \text { birrea (A. } & \text { Rich.) } \\
\text { Hochst.subsp. caffra (Sond.) Kokwaro } & \end{array}$ & Southern Africa \\
\hline & Spondias dulcis Sol. ex Parkinson & South-Eastern Asia \\
\hline & Spondias mombin L. & Afrotropical \\
\hline Annonaceae & Annona muricata $\mathrm{L}$. & South America \\
\hline & Annona reticulata $\mathrm{L}$. & Caribbean \\
\hline & Annona squamosa $\mathrm{L}$. & South America \\
\hline & Cananga odorata (Lam.) Hook.f. \&Thomson & South-Eastern Asia \\
\hline & Polyalthia longifolia (Sonn.) Thwaites & Southern Asia \\
\hline Apocynaceae & Adenium obesum (Forsskål) Roemer \& Schultes & Southern Africa \\
\hline & Allamanda blanchetii A. DC. & South America \\
\hline & Allamanda cathartica $\mathrm{L}$. & South America \\
\hline & Calotropis procera (Aiton) W.T. Aiton & Native \\
\hline & Cascabela thevetia $(\mathrm{L}$.$) Lippold$ & South America \\
\hline & Holarrhena floribunda (G.Don) T. Durand \& Schinz & Native \\
\hline & Landolphia heudelotii A.DC. & Native \\
\hline & Nerium oleander L. & Western Asia \\
\hline & Plumeria alba $\mathrm{L}$. & $\begin{array}{l}\text { Northern and South } \\
\text { America }\end{array}$ \\
\hline & Plumeria pudica Jacq. & $\begin{array}{l}\text { Central and South } \\
\text { America }\end{array}$ \\
\hline & Plumeria rubra L. & $\begin{array}{l}\text { Northern America } \\
\text { and Caribbean }\end{array}$ \\
\hline & Rauvolfia viridis Willd., ex Roemer \& Schultes & $\begin{array}{l}\text { Caribbean and South } \\
\text { America }\end{array}$ \\
\hline & Rauvolfia vomitoria Afzel. & Native \\
\hline & Saba senegalensis (A. DC.) Pichon & Native \\
\hline & Voacanga africana Stapf ex Scott-Elliot, 1894 & Native \\
\hline Araliaceae & Aralia balfouriana André & $\begin{array}{l}\text { South and Northern } \\
\text { America }\end{array}$ \\
\hline & Aralia filicifolia Ridl. & $\begin{array}{l}\text { South and Northern } \\
\text { America }\end{array}$ \\
\hline & Scheffleria arboricola (Hayata) Merr. & Southern Asia \\
\hline Asteraceae & Vernonia amygdalina Del. & Native \\
\hline & Vernonia colorata (Willd.) Drake & Native \\
\hline & Zinnia elegans $\mathrm{L}$. & Northern America \\
\hline Bignoniaceae & Crescentia cujete $\mathrm{L}$. & South America \\
\hline & Jacaranda mimosifolia D. Don & South America \\
\hline & Kigelia africana (Lam.) Benth. & Native \\
\hline & Newboulidia laevis (P.Beauv.) Seemann ex Bureau & Western Africa \\
\hline & Stereospermum kunthianum Cham. & Native \\
\hline
\end{tabular}




\begin{tabular}{|c|c|c|}
\hline & Tecoma stans Juss. (L.) Juss. ex Kunth & $\begin{array}{l}\text { Central and South } \\
\text { America }\end{array}$ \\
\hline Bixaceae & Bixa orellana $\mathrm{L}$. & South America \\
\hline \multirow[t]{2}{*}{ Boraginaceae } & Cordia myxa $\mathrm{L}$. & Asia and Australian \\
\hline & Cordia sebastiana $\mathrm{L}$. & South America \\
\hline Burseraceae & Boswellia dalzielli Hutch. & Native \\
\hline \multirow[t]{2}{*}{ Cactaceae } & Echinocactus grusonii Hildmann & South America \\
\hline & Opuntia ficus-indica (L.) Mill. & Central America \\
\hline \multirow[t]{4}{*}{ Capparaceae } & Boscia senegalensis (Pers.) Lam. ex Poir. & Native \\
\hline & Cadaba farionsa Forssk. & Native \\
\hline & Maerua angolensis DC. & Native \\
\hline & Maerua crassifolia Forssk. & Native \\
\hline Caricaceae & Carica papaya $\mathrm{L}$. & Central America \\
\hline Casuarinaceae & Casuarina equisetifolia $\mathrm{L}$. & $\begin{array}{l}\text { Australia and } \\
\text { Zealand }\end{array}$ \\
\hline \multirow[b]{5}{*}{ Combretaceae } & Anogeissus leiocarpa (DC.) Guill. \& Perr & Native \\
\hline & Combretum micranthum G. Don & Native \\
\hline & Quisqualis indica $\mathrm{L}$. & South-Eastern Asia \\
\hline & Terminalia catappa $\mathrm{L}$. & $\begin{array}{l}\text { Australia and } \\
\text { Zealand }\end{array}$ \\
\hline & Terminalia mantaly H. Perrier & Madagascan \\
\hline Convolvulaceae & Argyreia nervosa (Burm. f.) Bojer & Southern Asia \\
\hline Crassulaceae & $\begin{array}{l}\text { Kalanchoe daigremontianum Raymond Hamet \& H. } \\
\text { Perrier }\end{array}$ & Madagascan \\
\hline \multirow[t]{2}{*}{ Ebenaceae } & Diospyros kaki Thunb. & Southern Asia \\
\hline & Diospyros mespiliformis A. Rich. & Native \\
\hline \multirow[t]{14}{*}{ Euphhorbiaceae } & Acalypha hispidula Burm. f. & Southern Asia \\
\hline & Acalypha wilkesiana Muüll. Arg. & Southern Asia \\
\hline & Euphorbia basalmifera Aiton & Western Africa \\
\hline & Euphorbia caudelabrum Kotschy & Eastern Africa \\
\hline & Euphorbia ingens E.Mey. ex Boiss. & Southern Africa \\
\hline & Euphorbia kamerunica Pax & Central Africa \\
\hline & Euphorbia milii Des Moul. & Madagascan \\
\hline & Euphorbia pulcherrima Willd. ex Klotzsch & $\begin{array}{l}\text { Central and South } \\
\text { America }\end{array}$ \\
\hline & Euphorbia tirucalli $\mathrm{L}$. & Eastern Africa \\
\hline & Euphorbia tithymaloides L. & $\begin{array}{l}\text { Northern and } \\
\text { Central America }\end{array}$ \\
\hline & Hura crepitans $\mathrm{L}$. & South America \\
\hline & Jatropha curcas L. & South America \\
\hline & Jatropha gossypiifolia L. & South America \\
\hline & Jatropha integerrima Jacq. & Caribbean \\
\hline
\end{tabular}




\begin{tabular}{|c|c|c|}
\hline & Jatropha multifida $\mathrm{L}$. & Northern America \\
\hline & Jatropha podagrica Hook. & Central America \\
\hline & Olea europae L. & $\begin{array}{l}\text { Western Asia } \\
\text { Northern Africa }\end{array}$ \\
\hline & Ricinus communis L. & Native \\
\hline & Codiaeum variegatum (L.) Rumph. ex A. Juss. & South-Eastern Asia \\
\hline \multirow{14}{*}{$\begin{array}{l}\text { Fabaceae - } \\
\text { Caesalpinioideae }\end{array}$} & Afzelia africana $\mathrm{Sm}$. \& Pers. & Native \\
\hline & Bauhinia purpurea L. Benth. & $\begin{array}{l}\text { Southern and South- } \\
\text { Eastern Asia }\end{array}$ \\
\hline & Bauhinia rufescens Lam. & Native \\
\hline & Caesalpinia pulcherrima (L.) Sw. & Northern America \\
\hline & Cassia sieberiana DC. & Native \\
\hline & Cordyla pinnata (Lepr. ex A.Rich.) Milne-Redh. & Native \\
\hline & Delonix regia (Bojer ex Hook.) Raf. & Madagascan \\
\hline & Parkinsonia aculeata $\mathrm{L}$. & South America \\
\hline & Peltophorum ferrugineum (Decaisne) Bentha & South-Eastern Asia \\
\hline & Piliostigma reticulatum (DC.) Hochst. & Native \\
\hline & Senna alata (L.) Roxb. & Northern America \\
\hline & Senna siamea (Lam.) Irwin \& Barneby & South-Eastern Asia \\
\hline & Senna spectabilis (DC.) Irwin \& Barneby & $\begin{array}{l}\text { South and Northern } \\
\text { America }\end{array}$ \\
\hline & Tamarindus indica $\mathrm{L}$. & Native \\
\hline \multirow{9}{*}{\begin{tabular}{|l} 
Fabaceae \\
Faboideae
\end{tabular}} & Andira inermis (W.Wright) Kunth ex DC. & Central America \\
\hline & Dalbergia sissoo Roxb. ex DC. & Southern Asia \\
\hline & Erythrina indica Lam. & Southern Asia \\
\hline & Erythrina senegalensis DC. & Native \\
\hline & Erythrina sigmoïdea Hua & Native \\
\hline & Faidherbia albida (Del.) A. Chev. & Native \\
\hline & Gliciridia sepium (Jacq.) Kunth ex Walp. & $\begin{array}{l}\text { South and Central } \\
\text { America }\end{array}$ \\
\hline & Leucaena leucocephala (Lam.) de Wit & $\begin{array}{l}\text { South America, } \\
\text { Caribbean }\end{array}$ \\
\hline & Canavalia ensiformis ( L. ) DC. & $\begin{array}{l}\text { Africa, Central } \\
\text { America }\end{array}$ \\
\hline \multirow[t]{5}{*}{$\begin{array}{l}\text { Fabaceae } \\
\text { Mimosoideae }\end{array}$} & Acacia holosericea A. Cunn. ex G. Don & $\begin{array}{l}\text { Australia and } \\
\text { Zealand }\end{array}$ \\
\hline & Albizia chevalieri Harms & Native \\
\hline & Albizia julibrissin Durazz. & $\begin{array}{l}\text { Wester and southern } \\
\text { Asia }\end{array}$ \\
\hline & Albizia lebbeck (L.) Benth. & Southern Asia \\
\hline & Calliandra calothyrsus Meissner & Central America \\
\hline
\end{tabular}




\begin{tabular}{|c|c|c|}
\hline & Enterolobium cyclocarpum (Jacq.) Grise & $\begin{array}{l}\text { Caribbean and South } \\
\text { America }\end{array}$ \\
\hline & Parkia biglobosa (Jacq.) Benth. & Native \\
\hline & Pithecellobium dulce (Roxb.) Benth. & $\begin{array}{l}\text { Central, Northern } \\
\text { and South America }\end{array}$ \\
\hline & Prosopis africana (Guill. \& Perr.) Taub. & Native \\
\hline & Prosopis juliflora (Sw.) DC. & $\begin{array}{l}\text { Northern, South } \\
\text { America and } \\
\text { Caribbean }\end{array}$ \\
\hline & Senegalia erythrocalyx (Brenan) Kyal. \& Baotwr & Native \\
\hline & Senegalia senegal (L.) Britton \& P. Wilson & Native \\
\hline & Vachellia nilotica (L.) P.J. H. Hurter \& Mabb. & Native \\
\hline & \begin{tabular}{|l|l|} 
Vachellia seyal (Del.) P.J.H.Hurter \\
\end{tabular} & Native \\
\hline & Vachellia tortilis (Forssk.) Galasso \& Banfi & Native \\
\hline Hydrangeaceae & Hydrangea paniculata Siebold & South-Eastern Asia \\
\hline Lamiaceae & Clerodendrum inerme (L.) Gaertn & South-eastern Asia \\
\hline & Clerodendrum thomsoniae Balf. & Tropical Africa \\
\hline Lauraceae & Laurus nobilis $\mathrm{L}$. & Europe \\
\hline & Persea americana Mill. & $\begin{array}{l}\text { Central and } \\
\text { Northern America }\end{array}$ \\
\hline Lecythidaceae & Barringtonia asiatica (L.) Kurz & Southern Asia \\
\hline & Lagerstroemia indica $\mathrm{L}$. & South-Eastern Asia \\
\hline & Lawsonia inermis $\mathrm{L}$. & $\begin{array}{l}\text { Western Asia, } \\
\text { Northern Africa }\end{array}$ \\
\hline Lythraceae & Punica granatum $\mathrm{L}$. & Southern Asia \\
\hline Malvaceae & Abutilon hybridum Hort. ex Siebert. \& Voss & South America \\
\hline & Adansonia digitata $\mathrm{L}$. & Native \\
\hline & Bombax ceiba $\mathrm{L}$. & Southern Asia \\
\hline & Ceiba pentandra (L.) Gaertn. & $\begin{array}{l}\text { Central America, } \\
\text { South America }\end{array}$ \\
\hline & Ceiba speciosa A.St.-Hil. & South America \\
\hline & Dombeya cayeuxii André & $\begin{array}{l}\text { Tropical Africa and } \\
\text { Madagascan }\end{array}$ \\
\hline & Gossypium arboreum $\mathrm{L}$. & Asia \\
\hline & Gossypium barbadense $\mathrm{L}$. & South America \\
\hline & Grewia bicolor Juss. & Native \\
\hline & Grewia flavescens Juss. & Native \\
\hline & Hibiscus rosa-sinensis $\mathrm{L}$. & South-Eastern Asia \\
\hline Meliaceae & Azadirachta indica A. Juss. & Southern Asia \\
\hline & Khaya senegalensis (Desr.) A. Juss. & Native \\
\hline & Melia azedarach $\mathrm{L}$. & Southern Asia \\
\hline Moraceae & Artocarpus altilis (Parkinson) Fosberg & South-Eastern Asia \\
\hline & Artocarpus heterophyllus Lam. & Southern Asia \\
\hline
\end{tabular}




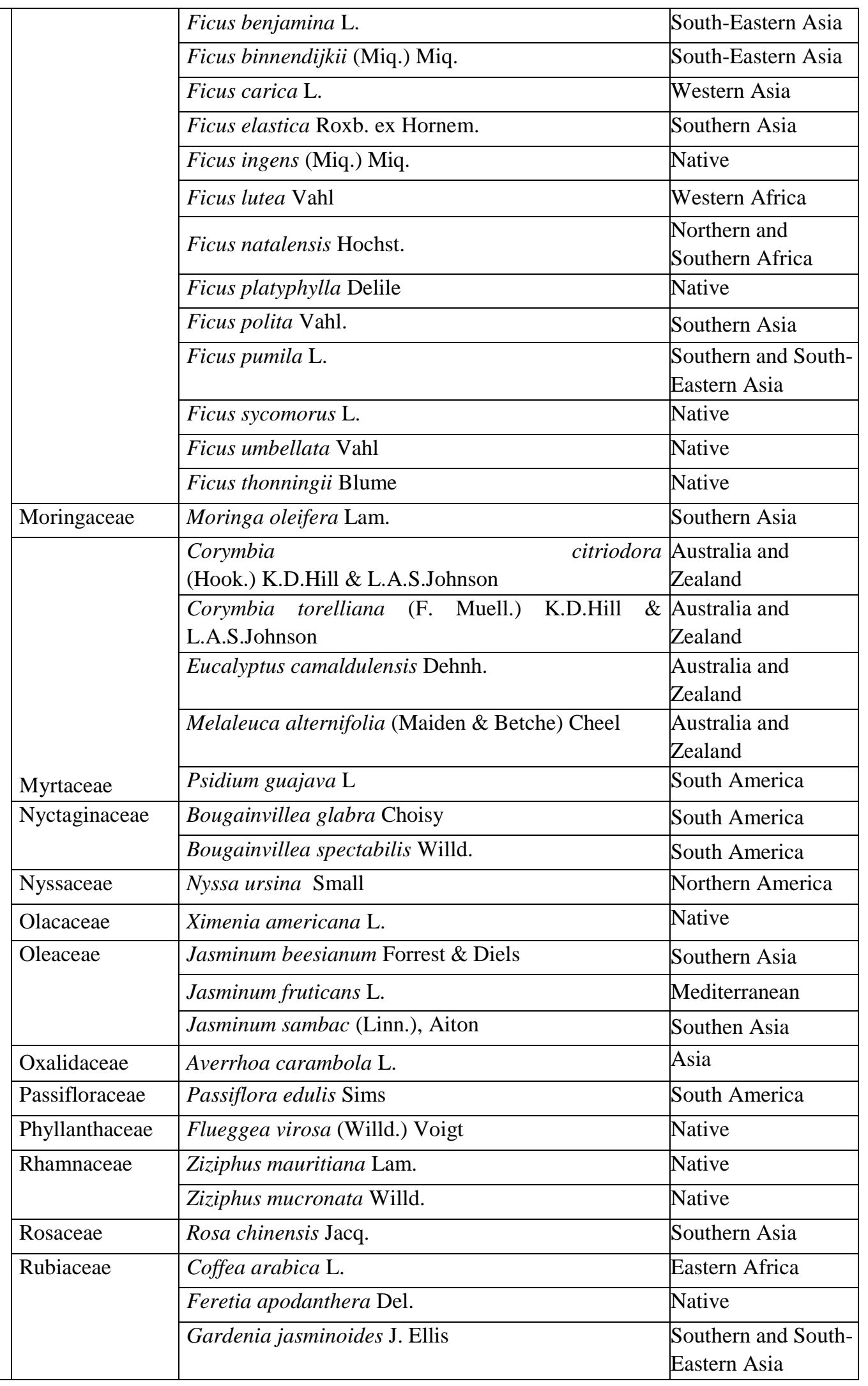




\begin{tabular}{|c|c|c|}
\hline & Ixora acuminata Roxb & South-Eastern Asia \\
\hline & Ixora chinensis Lam. & South-Eastern Asia \\
\hline & Ixora coccinea $\mathrm{L}$. & South-Eastern Asia \\
\hline & Ixora javanica (Blume) DC. & South-Eastern Asia \\
\hline & Ixora macrothyrsa (Teijsm. \& Binn.) R. E. Br. & Southern Asia \\
\hline & Ixora miniature & $\begin{array}{l}\text { Southern and South- } \\
\text { Eastern Asia }\end{array}$ \\
\hline & Morinda citrifolia $\mathrm{L}$. & Asia \\
\hline & Mussaenda philippica A. Rich. & South-Eastern Asia \\
\hline & Sarcocephalus latifolius (Smith) Bruce & Native \\
\hline \multirow[t]{9}{*}{ Rutaceae } & Citrus aurantiifolia (Christm.) Swingle & Southern Asia \\
\hline & \begin{tabular}{|l|} 
Citrus limon (L.) Burm. f. \\
\end{tabular} & South-eastern Asia \\
\hline & Citrus maxima (Burm.) Merr. & South-eastern Asia \\
\hline & Citrus reticulata Blanco & South-eastern Asia \\
\hline & Citrus sinensis (L.) Osbeck & South-eastern Asia \\
\hline & Citrus tangelo J.W.Ingram \& H.E. Moore & Southern Asia \\
\hline & Fortunella japonica (Thunb.) Swingle & South-Eastern Asia \\
\hline & Murraya paniculata (L.) Jack & South-Eastern Asia \\
\hline & Zanthoxylum zanthoxyloides (Lam.) Zepern.\&Timler & Native \\
\hline \multirow[b]{3}{*}{ Sapindaceae } & Blighia sapida K. D. Koenig & $\begin{array}{l}\text { Central and Western } \\
\text { Africa }\end{array}$ \\
\hline & Dodonaea viscosa Jacq. & $\begin{array}{l}\text { Australia and } \\
\text { Zealand }\end{array}$ \\
\hline & Melicoccus bijugatus Jacq. & $\begin{array}{l}\text { South America, } \\
\text { Caribbean }\end{array}$ \\
\hline \multirow[t]{2}{*}{ Sapotaceae } & Achras sapota $\mathrm{L}$. & Central America \\
\hline & Vitellaria paradoxa Gaertn. f. & Native \\
\hline \multirow[t]{5}{*}{ Sterculiaceae } & Cola cordifolia (Cav.) R. Br. & Native \\
\hline & Cola laurifolia Mast. & Western Africa \\
\hline & Cola nitida (Vent.) Schott \& Endl. & Western Africa \\
\hline & Sterculia setigera Delile & Native \\
\hline & Theobroma cacao L. & Northern America \\
\hline Strelitziaceae & Ravenala madagascariensis Sonn. & Madagascan \\
\hline Theaceae & Camelia japonica $\mathrm{L}$. & Northern America \\
\hline \multirow[b]{6}{*}{ Verbenaceae } & Aloysia citrodora Palau & Southern America \\
\hline & Duranta erecta $\mathrm{L}$. & $\begin{array}{l}\text { Central and South } \\
\text { America }\end{array}$ \\
\hline & Gmelina arborea Roxb. & Southern Asia \\
\hline & Lantana camara L. & South America \\
\hline & \begin{tabular}{|l|} 
Tectona grandis $\mathrm{L} . \mathrm{f}$. \\
\end{tabular} & South-Eastern Asia \\
\hline & Vitex doniana Sweet, 1827 & Native \\
\hline \multirow[t]{2}{*}{ Vitaceae } & Cissus quadrangularis $\mathrm{L}$ & Native \\
\hline & Vitis vinifera $\mathrm{L}$. & Mediterranean \\
\hline Zygophyllaceae & Balanites aegyptiaca (L.) Del. & Native \\
\hline
\end{tabular}




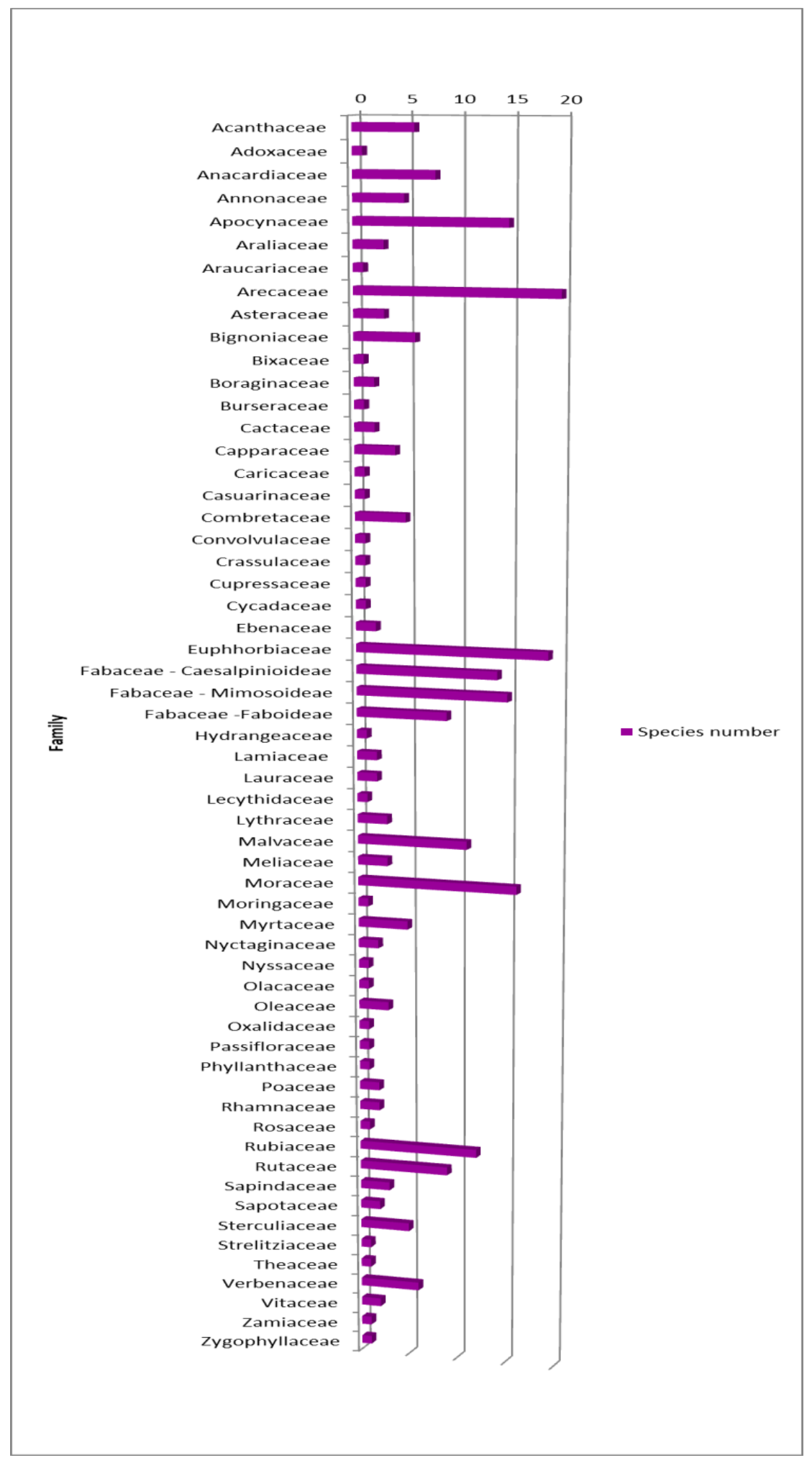

Figure 3: Floristic diversity of Burkina Faso city trees. 
Table 2: Characteristics of Burkina Faso's native urban trees.

\begin{tabular}{|c|c|c|c|}
\hline $\mathbf{N}^{\circ}$ & Family & Species name & Habitat \\
\hline$\overline{1}$ & Anacardiaceae & $\begin{array}{l}\text { Haematostaphis barteri } \\
\text { Hook.f. }\end{array}$ & $\begin{array}{l}\text { Sudan and Guinean savannahs on rocky hill soils } \\
\text { (Arbonnier, 2002; Akoègninou et al., 2006). }\end{array}$ \\
\hline 2 & & $\begin{array}{l}\text { Lannea microcarpa Engl. \& } \\
\text { K.Krause }\end{array}$ & $\begin{array}{l}\text { Sahel-Sudanese savannahs, on rocky soils and fresh and } \\
\text { deep compact soils (Arbonnier, 2002). }\end{array}$ \\
\hline 3 & & $\begin{array}{l}\text { Sclerocarya birrea (A. Rich.) } \\
\text { Hochst. subsp. birrea }\end{array}$ & $\begin{array}{l}\text { Sahel-Saharan savannahs, Sahel-Sudanese and Sudanese } \\
\text { areas, on sandy-textured soils (Lebrun et al., 1991; } \\
\text { Akoègninou et al., 2006). }\end{array}$ \\
\hline$\overline{4}$ & & Spondias mombin L. & $\begin{array}{l}\text { Sudan savannas and Guinean preforestry, gardens and } \\
\text { swamps (Arbonnier, 2002; Akoègninou et al., 2006). }\end{array}$ \\
\hline$\overline{5}$ & Apocynaceae & $\begin{array}{l}\text { Holarrhena floribunda } \\
\text { (G.Don) T.Durand \& Schinz }\end{array}$ & $\begin{array}{l}\text { Tree savannas and clear forests, Sudan areas in Guinea, } \\
\text { forest galleries and river banks (north), rocky hills } \\
\text { (south), wastelands and fallows (Arbonnier, 2002; } \\
\text { Akoègninou et al., 2006). }\end{array}$ \\
\hline 6 & & Landolphia heudelotii A.DC. & $\begin{array}{l}\text { Groves and wooded savannahs of the Sudanese and } \\
\text { Sudan-Guinean areas, on drained, sandy soils, on cracked } \\
\text { armour, ravines (Lebrun et al., 1991; Arbonnier, 2002). }\end{array}$ \\
\hline$\overline{7}$ & & Rauvolfia vomitoria Afzel. & $\begin{array}{l}\text { Forest galleries in the Sudan area, bushes and secondary } \\
\text { forests (Akoègninou et al., 2006). }\end{array}$ \\
\hline 8 & & $\begin{array}{l}\text { Saba senegalensis (A. DC.) } \\
\text { Pichon }\end{array}$ & $\begin{array}{l}\text { Gravel soils and armour in the Sudan area, termite } \\
\text { mounds (Lebrun et al., 1991). }\end{array}$ \\
\hline$\overline{9}$ & & $\begin{array}{ll}\text { Calotropis } & \text { procera } \\
\text { (Aiton) W.T. Aiton } & \\
\end{array}$ & $\begin{array}{l}\text { Sahelian species that prefers sandy soils, colonizes wetter } \\
\text { areas on degraded soils (Arbonnier, 2002). }\end{array}$ \\
\hline 10 & Arecaceae & Borassus aethiopum L. & $\begin{array}{l}\text { Temporarily flooded shallows in the Sahel-Sudanese and } \\
\text { Sudanese areas (Maydell, 1983) }\end{array}$ \\
\hline 11 & & $\begin{array}{l}\text { Borassus akeasii Bayton, } \\
\text { Ouédraogo \& Guinko }\end{array}$ & West African Endemic (Thiombiano et al., 2012) \\
\hline 12 & & Elaeis guineensis Jacq. & $\begin{array}{l}\text { Sudan-Guinean forests and savannahs in Guineans, on } \\
\text { well-drained and cool soils, on termite mounds in flood- } \\
\text { like shoals (Arbonnier, 2002) }\end{array}$ \\
\hline 13 & & Hyphaene thebaica (L.) Mart. & $\begin{array}{l}\text { Irregularly distributed species, often planted. Locally } \\
\text { abundant and gregarious (Arbonnier, 2002). }\end{array}$ \\
\hline 14 & & Raphia sudanica A. Chev. & $\begin{array}{l}\text { Irregularly distributed species, Sudanese savannahs and } \\
\text { forest galleries (Sacandé et al, 2012) }\end{array}$ \\
\hline 15 & Asteraceae & Vernonia amygdalina Del. & $\begin{array}{l}\text { Wet savannahs, backwaters, sometimes planted (César et } \\
\text { al., 2009). }\end{array}$ \\
\hline 16 & & $\begin{array}{l}\text { Vernonia colorata (Willd.) } \\
\text { Drake }\end{array}$ & $\begin{array}{l}\text { Sudan-Guinean and Guinean forests and savannahs, } \\
\text { rivers on fresh and drained soils, forest galleries, fallows, } \\
\text { banks of backwaters in the Sahelian zone (Lebrun et al., } \\
\text { 1991; Arbonnier, 2002; Akoègninou et al, 2006). }\end{array}$ \\
\hline$\overline{17}$ & Balanitaceae & Balanites aegyptiaca (L.) Del. & $\begin{array}{l}\text { Sahelian and Sudanese areas, soils eroded or trampled by } \\
\text { livestock (Lebrun et al., 1991). }\end{array}$ \\
\hline 18 & Bignoniaceae & $\begin{array}{l}\text { Kigelia africana (Lam.) } \\
\text { Benth. }\end{array}$ & $\begin{array}{l}\text { Sudan and Guinean forests and savannahs, thicketed, } \\
\text { forest galleries, usually on well-drained soils (Arbonnier, } \\
\text { 2002; Akoègninou et al, 2006). }\end{array}$ \\
\hline
\end{tabular}




\begin{tabular}{|c|c|c|c|}
\hline$\overline{19}$ & & $\begin{array}{l}\text { Stereospermum kunthianum } \\
\text { Cham. }\end{array}$ & $\begin{array}{l}\text { Tree savannas, forest galleries, fallows in Sahel- } \\
\text { Sudanese and Guinean areas, on all types of soils } \\
\text { (Arbonnier, 2002; Akoègninou et al, 2006). }\end{array}$ \\
\hline$\overline{20}$ & Boraginaceae & Cordia myxa $L$. & $\begin{array}{l}\text { Sudan-Guinean savannahs in Guineans, on cool, deep } \\
\text { soils, near rivers (Arbonnier, 2002). }\end{array}$ \\
\hline$\overline{21}$ & Burseraceae & Boswellia dalzielli Hutch. & $\begin{array}{l}\text { Sahel-Sudanese savannahs in Sudan-Guineans with a } \\
\text { very marked dry season, on dry soils, often on hills or } \\
\text { granite rocky outcrops (Arbonnier, 2002). }\end{array}$ \\
\hline$\overline{22}$ & Capparaceae & $\begin{array}{l}\text { Boscia senegalensis (Pers.) } \\
\text { Lam. ex Poir. }\end{array}$ & $\begin{array}{l}\text { Dry stations, rocky, lasteritical, sandy-clay compacts and } \\
\text { old termite mounds (Lebrun et al., 1991; Arbonnier, } \\
\text { 2002). }\end{array}$ \\
\hline$\overline{23}$ & & Cadaba farionsa Forssk. & $\begin{array}{l}\text { Dry and arid land, especially at the foot of termite } \\
\text { mounds (Lebrun et al., 1991). }\end{array}$ \\
\hline$\overline{24}$ & & Maerua angolensis DC. & $\begin{array}{l}\text { Savannahs of the South-Sahelian and Northern Sudanese } \\
\text { areas on sandy soils (Lebrun et al., 1991; Arbonnier, } \\
\text { 2002; Akoègninou et al., 2006). }\end{array}$ \\
\hline$\overline{25}$ & & ifolia Forssk. & $\begin{array}{l}\text { Sahelo-Sudanian and Sudanese dry savannahs on sandy } \\
\text { soils (Arbonnier, 2002). }\end{array}$ \\
\hline
\end{tabular}

26 Combretaceae Anogeissus leiocarpa (DC.) Forest galleries, dry forests, savannahs in the SudanGuill. \& Perr Sahelian to The Sudan-Guinean zone, on generally compact soils (Arbonnier, 2002).

27 Combretum micranthum G. Sahelian skeletal soils, on gravel in the Sudan area, Don almost impervious degraded termite mounds (Lebrun et al., 1991; Thiombiano, 2005).

28 Ebenaceae Diospyros mespiliformis A. Dense forests, clear forests, forest galleries and Rich. $\quad$ savannahs, near the marigots (Lebrun et al., 1991; Akoègninou et al., 2006).

29 Euphorbiaceae Euphorbia balsamifera Aiton Sahelian and Sudanese savannahs, on sandy soils or rocky stations (Arbonnier, 2002).

\begin{tabular}{|c|c|c|c|}
\hline \multirow{2}{*}{\multicolumn{2}{|c|}{30}} & & \\
\hline & & Ricinus communis $\mathrm{L}$. & $\begin{array}{l}\text { Proximity to dams and gardens, storm stations (Lebrun et } \\
\text { al., 1991; Akoègninou et al., 2006). }\end{array}$ \\
\hline 31 & \multirow{6}{*}{$\begin{array}{l}\text { Fabaceae - } \\
\text { Caesalpinioidea } \\
\text { e }\end{array}$} & Piliostigma reticulatum (DC.) & Dry savannahs, on wet sandy soils (Lebrun et al., 1991; \\
\hline & & Hochst. & Akoègninou et al., 2006). \\
\hline 32 & & Afzelia africana Sm. \& Pers. & $\begin{array}{l}\text { Sudan's savannahs, forest galleries, Guinean forests, on } \\
\text { deep sandy and alluvial soils (Lebrun et al., 1991; } \\
\text { Arbonnier, 2002). }\end{array}$ \\
\hline 33 & & Bauhinia rufescens Lam. & $\begin{array}{l}\text { Wet sandy soils in the Sahelian zone (Lebrun et al., } \\
\text { 1991). }\end{array}$ \\
\hline 34 & & Cassia sieberiana DC. & $\begin{array}{l}\text { Sudan-Guinean and Sudanese savannahs, forest galleries } \\
\text { on all types of soils, preferably laterite soils (Lebrun et } \\
\text { al., 1991; Arbonnier, 2002). }\end{array}$ \\
\hline$\overline{35}$ & & Tamarindus indica $\mathrm{L}$. & $\begin{array}{l}\text { Savannahs, often on termite mounds (Akoègninou et al., } \\
\text { 2006). }\end{array}$ \\
\hline 36 & \multirow[t]{3}{*}{$\begin{array}{l}\text { Fabaceae- } \\
\text { Faboideae }\end{array}$} & $\begin{array}{l}\text { Andira inermis (W.Wright) } \\
\text { DC. }\end{array}$ & $\begin{array}{l}\text { Lowlands, banks of marigots on deep soils (Lebrun et al., } \\
\text { 1991; Arbonnier, 2002). }\end{array}$ \\
\hline$\overline{37}$ & & $\begin{array}{l}\text { Cordyla pinnata (Lepr. ex } \\
\text { A.Rich.) Milne-Redh. }\end{array}$ & $\begin{array}{l}\text { Sudan's savannahs, dry forests, on medium soils } \\
\text { (Arbonnier, 2002). }\end{array}$ \\
\hline 38 & & Erythrina senegalensis DC. & $\begin{array}{l}\text { Savannahs on sandy colluvions (Berhaut, 1974; Lebrun } \\
\text { et al., 1991; Akoègninou et al., 2006). }\end{array}$ \\
\hline
\end{tabular}




\begin{tabular}{|c|c|c|c|}
\hline 39 & & $\begin{array}{l}\text { Erythrina } \\
\text { (introduit) }\end{array}$ & $\begin{array}{l}\text { Tree savannas and clear forests, forest galleries, riparian } \\
\text { forests (César et al., 2009). }\end{array}$ \\
\hline$\overline{40}$ & \multirow[t]{9}{*}{$\begin{array}{l}\text { Fabaceae- } \\
\text { Mimosoideae }\end{array}$} & Albizia chevalieri Harms & $\begin{array}{l}\text { Alluvial terraces and wet savannahs in the Sudan area } \\
\text { (Lebrun et al., 1991). }\end{array}$ \\
\hline$\overline{41}$ & & $\begin{array}{l}\text { Faidherbia albida (Del.) A. } \\
\text { Chev. }\end{array}$ & Savannahs, cultivated fields (Akoègninou et al., 2006). \\
\hline$\overline{42}$ & & $\begin{array}{lll}\text { Parkia biglobosa } & \text { (Jacq.) } \\
\text { Benth. } & & \\
\end{array}$ & $\begin{array}{l}\text { Tree savannas, lowlands and Sudanese savannahs } \\
\text { (Lebrun et al., 1991; Akoègninou et al, 2006). }\end{array}$ \\
\hline$\overline{43}$ & & $\begin{array}{l}\text { Prosopis africana (Guill. \& } \\
\text { Perr.) Taub. }\end{array}$ & $\begin{array}{l}\begin{array}{l}\text { Tree savannas, inselbergs, lowlands of Sudan } \\
\text { (Akoègninou et al., 2006). }\end{array} \\
\end{array}$ \\
\hline$\overline{44}$ & & $\begin{array}{l}\text { Senegalia erythrocalyx } \\
\text { (Brenan) Kyal. \& Baotwr }\end{array}$ & $\begin{array}{l}\text { Wet dense forests, pond banks on clay soils and gravel } \\
\text { armour, on termite mounds (Lebrun et al., 1991; } \\
\text { Arbonnier, 2002; Akoègninou et al, 2006). }\end{array}$ \\
\hline$\overline{45}$ & & $\begin{array}{l}\text { Senegalia senegal }(\mathrm{L} .) \text { Britton } \\
\& \mathrm{P} \text {. Wilson }\end{array}$ & $\begin{array}{l}\text { Sahelian Africa and the Arabian Peninsula (Ganaba, } \\
\text { 2008) }\end{array}$ \\
\hline$\overline{46}$ & & $\begin{array}{l}\text { Vachellia nilotica (L.) P.J. H. } \\
\text { Hurter \& Mabb. }\end{array}$ & $\begin{array}{l}\text { Sahel-Sudan region, on wet and heavy soils (Lebrun et } \\
\text { al., 1991; Arbonnier, 2002, Ganaba, 2008). }\end{array}$ \\
\hline$\overline{47}$ & & $\begin{array}{lll}\text { Vachellia seyal } & \text { (Del.) } \\
\text { P.J.H.Hurter } & & \\
\end{array}$ & $\begin{array}{l}\text { Sahel and Sudanian silty-clayey lowlands, silty-clayey } \\
\text { glazes (Lebrun et al., 1991, Ganaba 2008). }\end{array}$ \\
\hline 48 & & $\begin{array}{l}\text { Vachellia tortilis (Forssk.) } \\
\text { Galasso \& Banfi }\end{array}$ & $\begin{array}{l}\text { Sahelian zone, on sandy or slightly gravel and Saharan } \\
\text { glacis (Lebrun et al., 1991, Ganaba, 2008). }\end{array}$ \\
\hline 49 & \multirow[t]{6}{*}{ Malvaceae } & Adansonia digitata $\mathrm{L}$. & $\begin{array}{l}\text { Sahel-Sudan zone, on soils of varying quality, usually on } \\
\text { degraded termite mounds (Arbonnier, 2002). }\end{array}$ \\
\hline 50 & & Cola cordifolia (Cav.) R. Br. & $\begin{array}{l}\text { Sudan's savannahs and dry forests in Guinea, tolerates } \\
\text { temporary flooded soils (Arbonnier, 2002). }\end{array}$ \\
\hline 51 & & Cola laurifolia Mast. & $\begin{array}{l}\text { Banks of the great Sudanese marigots, on temporarily } \\
\text { flooded soils (Lebrun et al., 1991; Arbonnier, 2002). }\end{array}$ \\
\hline 52 & & Grewia bicolor Juss. & $\begin{array}{l}\text { Sahelo-Sudanese savannahs, at the edges of ponds, on } \\
\text { rocks, cracked lateritic armour and gravel soils } \\
\text { (Arbonnier, 2002). }\end{array}$ \\
\hline 53 & & Grewia flavescens Juss. & $\begin{array}{l}\text { Sahel-Sudanese savannahs, on various wet soils in the } \\
\text { rainy season (Arbonnier, 2002). }\end{array}$ \\
\hline 54 & & Sterculia setigera Delile & $\begin{array}{l}\text { Dry savannahs, on sandy or gravel soils (Lebrun et al., } \\
\text { 1991) }\end{array}$ \\
\hline 55 & Meliaceae & $\begin{array}{l}\text { Khaya senegalensis (Desr.) A. } \\
\text { Juss. }\end{array}$ & $\begin{array}{l}\text { Sudan's savannahs in Guineans, on deep, well-drained } \\
\text { soils (Arbonnier, 2002). }\end{array}$ \\
\hline 56 & \multirow[t]{6}{*}{ Moraceae } & Ficus abutilifolia (Miq.) Miq. & $\begin{array}{l}\text { Gallery forests and savannahs of Sahel-Sudanian areas in } \\
\text { Sudan-Guinea, rocky hills, rocky places in Burkina Faso } \\
\text { (Lebrun et al., 1991; Arbonnier, 2002). }\end{array}$ \\
\hline 57 & & Ficus ingens (Miq.) Miq. & $\begin{array}{l}\text { Savannahs in Sudanese areas, on sands and gravel } \\
\text { (Lebrun et al., 1991; Arbonnier, } 2002\end{array}$ \\
\hline 58 & & Ficus natalensis Hochst. & $\begin{array}{l}\text { Guinean savannahs and forest galleries, along the river, } \\
\text { wet and marshy stations (Arbonnier, 2002; Akoègninou } \\
\text { et al, 2006). }\end{array}$ \\
\hline 59 & & Ficus platyphylla Delile & $\begin{array}{l}\text { Lowlands in the Sudan area, savannahs, fallows (Lebrun } \\
\text { et al., 1991; Akoègninou et al., 2006). }\end{array}$ \\
\hline 60 & & Ficus sycomorus L. & Sandy lowlands in sub-Sahelian and Sudanese areas. \\
\hline 61 & & Ficus thonningii Blume & $\begin{array}{l}\text { Savannahs, Sudan-Sahelian forest galleries in Guineans, } \\
\text { thicketed (Arbonnier, 2002; Akoègninou et al., 2006). }\end{array}$ \\
\hline
\end{tabular}




\begin{tabular}{|c|c|c|c|}
\hline 62 & Phyllanthaceae & $\begin{array}{l}\text { Flueggea virosa } \quad \text { (Willd.) } \\
\text { Voigt }\end{array}$ & $\begin{array}{l}\text { Disturbed soils and fallows in Sahelinan savannahs in } \\
\text { Guineans, wet station in the Sahel, thicketed (Arbonnier, } \\
\text { 2002; Akoègninou et al., 2006). }\end{array}$ \\
\hline 63 & Poaceae & $\begin{array}{l}\text { Oxytenanthera abyssinica } \\
\text { (A.Rich.) Munro }\end{array}$ & $\begin{array}{l}\text { Eboulis of armour, forest galleries of wooded savannahs, } \\
\text { also planted in villages (Lebrun et al., 1991; Akoègninou } \\
\text { et al, 2006). }\end{array}$ \\
\hline$\overline{64}$ & Rhamnaceae & Ziziphus mauritiana Lam. & $\begin{array}{l}\text { Savannahs, sandy soils in Sahelian and Sahelian areas } \\
\text { (Lebrun et al., 1991). }\end{array}$ \\
\hline 65 & & Ziziphus mucronata Willd. & $\begin{array}{l}\text { Shrub savannas, fallows, gallery forests, shoals and } \\
\text { marigots in the Sahel-Sudan area (Lebrun et al., 1991; } \\
\text { Akoègninou et al., 2006) }\end{array}$ \\
\hline$\overline{66}$ & Rubiaceae & Feretia apodanthera Del. & $\begin{array}{l}\text { Berges of Subsahelian and Sudanese marigots, on termite } \\
\text { mounds, compact and battleships (Lebrun et al., 1991; } \\
\text { Arbonnier, 2002). }\end{array}$ \\
\hline 67 & & $\begin{array}{l}\text { Sarcocephalus } \\
\text { (Smith) Bruce }\end{array}$ & $\begin{array}{l}\text { Forest galleries, tree savannas, ponds and shallows of } \\
\text { Sudan-Guinean and Guinean savannahs, on more or less } \\
\text { well-drained wet soils (Arbonnier, 2002; Akoègninou et } \\
\text { al., 2006). }\end{array}$ \\
\hline$\overline{68}$ & Rutaceae & $\begin{array}{l}\text { Zanthoxylum zanthoxyloides } \\
\text { (Lam.) Zepern. \&Timler }\end{array}$ & $\begin{array}{l}\text { Groves and forest galleries of the Sudan-Guinean areas, } \\
\text { Guinean savannahs, on drained soils, termite mounds } \\
\text { (Lebrun et al., 1991; Arbonnier, 2002). }\end{array}$ \\
\hline 69 & Sapotaceae & Vitellaria paradoxa Gaertn. f. & $\begin{array}{l}\text { Sudanian savannahs in Guineans, on clay-clay soils with } \\
\text { clay-silical soils, decomposed lasterite terrain (Lebrun et } \\
\text { al., 1991; Akoègninou et al., 2006). }\end{array}$ \\
\hline 70 & Verbenaceae & Vitex doniana Sweet & $\begin{array}{l}\text { Savannahs, shallows and moist soils in the Sahelian area } \\
\text { (Lebrun et al., 1991; Akoègninou et al, 2006). }\end{array}$ \\
\hline 71 & & $\begin{array}{l}\text { Voacanga africana Stapf ex } \\
\text { Scott-Elliot }\end{array}$ & $\begin{array}{l}\text { Sudano guinean and mainland tropical Africa } \\
\text { (Arbonnier, 2002) }\end{array}$ \\
\hline$\overline{72}$ & Vitaceae & Cissus quadrangularis $\mathrm{L}$ & $\begin{array}{l}\text { Groves and ponds, Sahelian savannahs to Sudanese } \\
\text { (Arbonnier, 2002). }\end{array}$ \\
\hline
\end{tabular}

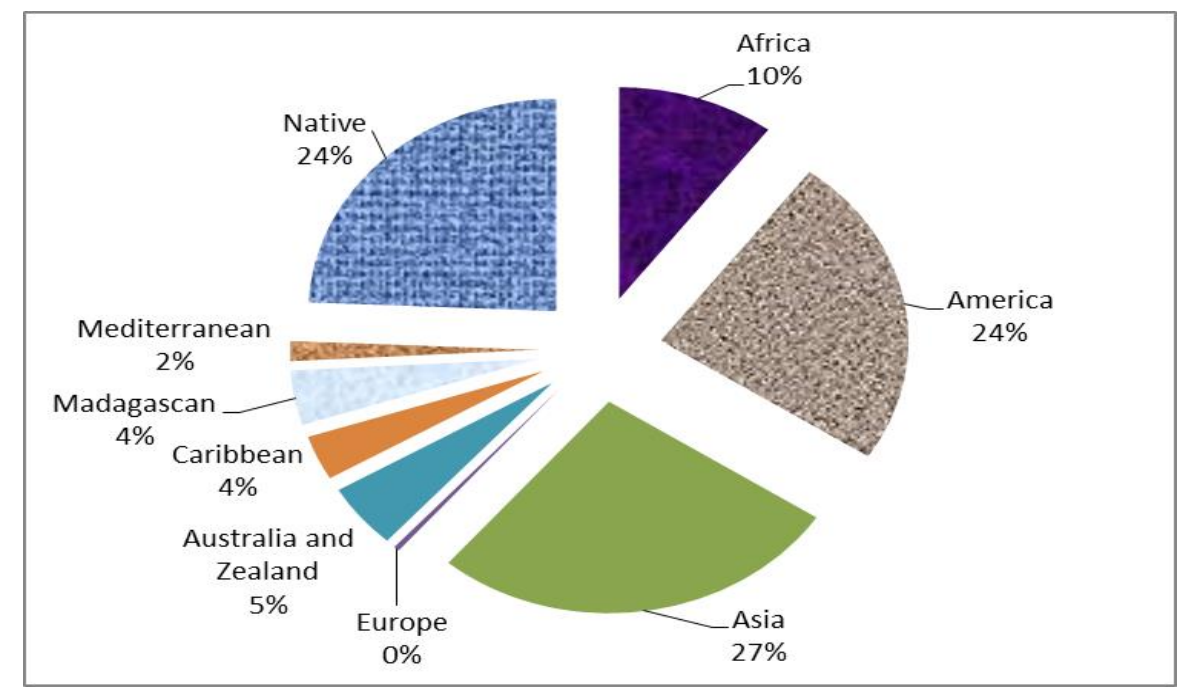

Figure 4 : Distribution of the origins of Burkina Faso urban trees. 


\section{DISCUSSION}

\section{Floristic diversity}

The flora of the city trees includes 251 species compared to the national flora which makes a total of 2067 species Thiombiano et al. (2012) and the ratio was 509 introduced species for 3451 natives in Togo (Radji et al., 2010). However, 36 introduced species not taken into account have been identified in urban flora. They are: Allamanda blanchetii, Aloysia citrodora Palau, Aralia balfouriana André, A. filicifolia Ridl., Araucaria heterophylla (Salisb.) Franco, Bismarkia nobilis Hildebrandt \& H. Wendl., Chamaedora elegans Mart., Chamaerops humilis L., Corymbia citriodora

(Hook.) K.D.Hill \& L.A.S.Johnson,

Dendrocalamus giganteus Wall ex. Munro, Diospyros kaki Thunb., Dypsis madagascariensis (Becc.) Beentje et $\mathrm{J}$. Dransf., Encephalartos woodii Sander, Euphorbia caudelabrum Kotschy, E. ingens E.Mey. ex Boiss., E. kamerunica Pax, E. milii Des Moul., E. tithymaloides L., Hydrangea paniculata Siebold, Hyophorbe lagenicaulis (L.H.Bailey) H.E.Moor, Ixora acuminate Roxb, I. chinensis Lam., I. coccinea L., I. macrothyrsa (Teijsm. \& Binn.) R. E. Br., Jatropha multifidi L., J. podagrica Hook., Morinda citrifolia L., Mussaenda philippica A.Rich., Olea europae L., Opuntia ficus-indica (L.) Mill., Plumeria pudica Jacq., Rauvolfia viridis Willd., ex Roemer \& Schultes, Rhopalostylis

(Sol. ex G.Forst.) H.Wendl. \& sapida

Roystonea regia (Kunth) O.F.Cook, Scheffleria arboricola (Hayata) Merr. and Spondias dulcis Sol. ex Parkinson.

The richness and diversity of species in this study are superior to those of some African cities studied such as Niamey which has 112 woody species belonging to 88 genera with 37 families and Maradi with 111 woody species belonging to 37 genera, with 34 families (Moussa et al., 2019), 109 ornamental species recorded in Dakar (Dieng et al., 2019) and 176 species reported in Kumassi (Nero et al., 2018), 132 species distributed in 95 genera and 32 families in Ziguinchor (Charahabil et al., 2018), Brazzaville 43 species belonging to 33 genera and 20 families in the sites studied, plus
28 other species in relics of forests located around the city (N'Zala and Miankodila, 2002). Our results are and lower than the 297 species of trees in Lomé in Togo (Raoufou and Kokou, 2013).

As in Burkina Faso, the ornamental flora of Dakar is dominated by Angiosperms which represent $84.61 \%$ of families and Gymnosperms (10.26\%) (Araucariaceae, Cupressaceae, Cycadaceae and Zamiaceae) (Dieng et al., 2019). On the other hand, the horticultural flora in Togo's cities is rich with 612 woody and herbaceous species, including 20 Pteridophytes, 17 Gymnosperms and 575 Angiosperms with more than $82 \%$ which are exotic to Africa (Raoufou and Kokou, 2013).

We found that Khaya senegalensis (African mahogany), Mangifera indica (mango tree) and Azadirachta indica (neem) were the most common species in cities: mango in southern Sudanese cities, African mahogany in northern and southern Sudan, and neem in the Sahelian ones. The last species, introduced from Ghana to Burkina Faso around 1934-1940 (Guinko, 2013; Ganaba, 2000) is also one of the most represented species in the flora of the green spaces of Lomé (45.23\%) according to Radji et al. (2010) and Polorigni et al. (2015). The most dominant species (based on density) in Niamey in Niger, are Azadirachta indica (neem), Balanites aegyptiaca (Thorn tree) and Terminalia mantaly (Umbrella tree), (Moussa et al., 2019). The five most common species in the city of Zinguinchor in Senegal are Azadirachta indica with a relative frequency of $90 \%$ followed by Mangifera indica (69\%), Elaeis guineensis (55\%), Adansonia digitata (52\%) and Borassus aethiopum (45\%) according to Charahabil et al. (2018). The most importance value index is for Gmelina arborea (5.82\%), Azadirachta indica (3.96\%) and Khaya senegalensis (3.74\%) in Abuja (Agbelade et al., 2016).

Azadirachta indica and Mangifera indica are the most dominant species in the northwestern Nigerian cities of Sokoto and Zaria (Dangulla et al., 2020) and the five most common species are Azadirachta indica with a relative frequency of $90 \%$ followed by Mangifera indica (69\%), Elaeis guineensis (55\%), Adansonia digitata (52\%) and Borassus 
aethiopum (45\%) in Ziguinchor according to Charahabil et al. (2018). In valleys in periurban post-conflict zone of Ziguinchor, were dominated to $70 \%$ by Eleais guineensis (Dasylva et al., 2017).

Our results indicate that out of the 251 species recorded, 215 species have already been cited in the updated flora of Burkina Faso (Thiombiano et al., 2012), while the 36 others have not been recorded and are among the species whose introductions continue to this day. For an updated total of 3003 species of the flora of Burkina Faso, urban floristic diversity represents for $8.36 \%$ of the country's flora.

\section{Origin of Burkina Faso City Trees}

Our results corroborate with those of Soma (2012), which found $86.17 \%$ exotic species and only $13.83 \%$ local species out of 188 ornamental species in nurseries in Ouagadougou. African cities are highly diverse in both native and exotic tree species but the exotic species dominate in many areas (Dangulla and Latifah, 2019). The low representativeness of local plants in ornamental choices is due to ecological and cultural reasons: their low productivity and the relative disinterest of customers (Soma, 2012). In the process of appropriation of urban space, local or spontaneous plants were undesirable and systematically eliminated from domestic and public gardens to make way for exotic ornamental plants, indicatives of cleanliness and order (Menozzi, 1998, 2007; Traoré, 2011). It was mainly Western residents of favored neighborhoods such as the "Zone du Bois" in Zogona and Petit Paris in Gounghin who maintained local species or bought them from local nurseries to decorate their living environment (Korbéogo, 2016).

The difference in climate between Europe and the Sahel may explain the low transfer of species of European and Mediterranean origin, despite colonial ties and rapprochement. The sharing of phylogenetic resources is limited to species such as Phoenix dactylifera (date palm). On the other hand, since the tropical zone is shared between Africa, South America, South Asia, Madagascar and Australia, the acclimatization and survival of the introduced plants is relatively easy and justifies their predominance.

Historically, many testimonies of the elderly indicate that the African mahoganies in our cities would have been planted just after the First World War to provide shade. Thus, the surrounding villages would take turns to send valid arms for planting and maintenance by watering them for a week. This was done in colonial cities such as Ouagadougou, as part of forced labour, as the colonial administration ordered the creation of protected forest areas such as the "Bois de Boulogne" in France, nowadays called Bangr-Weoogo Urban Park (meaning the forest of knowledge) in the middle of the city (Gnoumou et al., 2008).

\section{City Tree Constraints}

Urban forests forming ecosystems are more viable than isolated, often monospecific plantations. Moreover, trees in the cities are under enormous pressure, limiting their growth, functions and the ecosystem services. It can pose a threat hazards to infrastructure if not managed properly through tree falls, root incrustations in pipes and building foundations of that crack walls and overhead breaks in electrical and/or telephones wires. A rooting radius twice as large as the tree crown is required to allow the tree to remain stable against the elements. Otherwise, regular pruning of the canopy is required, which reduces the tree's operation and services.

In addition, lack of water is a major factor for urban trees. They can survive increased heat and insect pests unless they are not thirsty according to Meineke and Frank (2018).

\section{Prospects for City Trees}

In the city, planting trees is not enough. In the face of rising temperatures, or heat waves, planting trees is not enough to make cities more viable. The services rendered depend on what and where you plant and therefore on the parameters of the environment and how you plant so as not to uproot them shortly afterwards for development. Thus controversy has arisen over the felling of century-old trees in the city of Sya (Bobo Dioulasso) as part of the celebration of the fiftieth anniversary of Burkina Faso's independence in 2010 as part of 
the redevelopment of President Thomas Sankara's avenue in Ouagadougou in 2016. However, the dysfunctioning and failures related to the institutional and legal framework could explain the disappearing of the green spaces and reduction of urban trees number according to Polorigni et al. (2015).

Municipalities must then work to maintain and improve this potential through urban forest plantations that are more stable urban forest ecosystems than isolated plantations. The high richness of exotic trees in tropical urban green spaces could be a constraint. However, exotic trees pose a risk to native species and threaten urban and natural ecosystems through biological invasion and harm the maintenance of biodiversity. The native vegetation has an advantage over exotics species in ameliorating the urban heat effect as well as in their ability to cope with heat stress (Aguiar, 2012). Therefore, native or indigenous plants should be the first choice in tropical urban ecosystems (De Souza et al., 2020). In addition, it makes sense to plant fruit trees to endure the edible and resilient city. It was called "urban food forestry" (UFF) by Kyle and Kimberly (2013). Urban fruit trees could have diverse and direct socio-environmental impacts according to Colinas et al. (2019). However natural formations abound with many potentially ornamental species such as Bombax costatum Pellegr. \& Vuill., Gardenia ternifolia Schum. \& Thonn. (Korbéogo, 2016), Carissa edulis Vahl, Cochlospermum planchonii Hook.f, Combretum collinum Fresen., Combretum nigricans Lepr., Lannea acida Rich, Ozoroa insignis Kuntze, Parinari curatellifolia Planch, (Amani et al., 2019), Pterocarpus erinaceus Poir., Pterocarpus lucens Lepr., Dichrostachys cinerea (L.) Wight \& Arn., Pergularia tomentosa L., Strophantus hispidus DC., etc.

Native trees must be restored to strengthen the resilience of African cities.

\section{Conclusion}

This study identified 251 species of tree trees in cities from urban municipalities. This flora comprises 174 genera in 58 families. It is made up of $5.9 \%$ of Monocotyledons (2 families) $1.6 \%$ of Gymnosperms (4 families) and $92.5 \%$ of Dicotyledons (52 families). There are also 178 introduced species and 73 native species. The urban trees come from Africa (34\%)
Asia (27\%) America (24\%) Australia (5\%) Caribbean (3\% and Madagascar (4\%), Europe and the Mediterranean basin less than 2\%. All of these city trees are used as trees for alignments, courtyard and service gardens, parks and various green facilities. They provide various ecosystem services, including contributing to the fixation of urban dust from greenhouse gases from vehicles and combustions, reducing hot dry season heat waves, regulating runoff, maintaining various socio-economic and cultural activities in a word improve the living environment of city dwellers. The regressive evolution of the average global urban forest cover is drawing the attention of municipalities to build stable urban forest ecosystems by promoting native species and fruit trees in cities.

\section{COMPETING INTERESTS}

The author states that there is no competing interests for this article.

\section{AUTHORS' CONTRIBUTIONS}

SG collected the data and wrote the manuscript of this article.

\section{ACKNOWLEDGMENTS}

We thank Dr. Mamounata Belem/Ouédraogo and Mr. Diallo Adama for their help in determining certain species. Our recognition also goes to $\mathrm{Dr}$ Pascaline Coulibaly/Lingani, Dr Soungalo Soulama and anonymous readers for their contribution to improve the paper.

This research did not receive any specific grant from funding agencies in the public, commercial, or not-for-profit sectors.

\section{REFERENCES}

Agbelade AD, Onyekwelu JC, Oyun MB. 2016. Tree Species Diversity and their Benefits in Urban and Peri-Urban Areas of Abuja and Minna, Nigeria. Applied Tropical Agriculture, 21(3): 27-36.

Aguiar AC. 2012. Urban heat islands: differentiating between the benefits and drawbacks of using native and exotic vegetation in mitigating climate, Master of Science. Research thesis, School of Biological sciences, University of Wollongong, p.82.

Akoègninou $\mathrm{A}$, van der Burg $\mathrm{WJ}$, van der Maesen LJG. 2006. Flore Analytique du 
Bénin. Backhuys Publishers: Wageningen.

Amani KDSS, Kouassi FA, Ablan Aké-Assi E. 2019. Diversité floristique des plantes à potentialité décorative issues des formations naturelles du Nord de la Côte d'Ivoire. ESJ, 15(15): 46-63. DOI: 10.19044/esj. 2019.v15n15p46

Arbonnier M. 2002. Arbres, Arbustes et Lianes des Zones Sèches d'Afrique de l'Ouest (2nd edn). CIRAD-MNHN: Montpellier, Paris.

Charahabil MM, César B, Hamadou B, Ndiaye S, Diatta M. 2018. Diversité et structure des espaces végétalisés urbains de la ville de Ziguinchor, Sénégal. Int. J. Biol. Chem. Sci., 12(4): 1650-1666. DOI: https://dx.doi.org/10.4314/ijbcs.v12i4.12

Çay RD. 2016. Recreation and Urban Park Management. In Environment and Ecology at the Beginning of 21st Century, Efe R, Bızzarrı C (ed). St. Kliment Ohridski University Press: Cürebal \& GN Nyusupova; 302-312.

Colinas J, Bush P, Manaugh K. 2019. The socio-environmental impacts of public urban fruit trees: A Montreal case-study. Urban Forestry \& Urban Greening, 45: 126-132.

DOI: https://doi.org/10.1016/j.ufug.2018.05.002.

Dangulla M, Latifah AM. 2019. Progress and Methodological Approaches in Urban Trees and Forests Research in Africa. Journal of Environment and Earth Science, 9(9): 2224-3216. DOI: 10.7176/JEES/9-9-05

Dangulla M, Manaf LA, Ramli MF, Yacob MR. 2019. Urban tree composition, diversity and structural characteristics in North-western Nigeria. Urban Forestry \& Urban Greening, 48: 126512. DOI: https://doi.org/10.1016/j.ufug.2019.1265 12

Dieng B, Mbaye MS, Mballo R, Diouf M, Diouf J, Diouf N, Gueye FK, Ka SL, Sydibe M, Camara AA, Noba K. 2019. Caractérisation de la flore ornementale de la région de Dakar (Sénégal). J. Appl. Biosci., 138: 14029-14041. DOI: https://dx.doi.org/10.4314/jab.v138i1.3

Fuwape JA, Onyekwelu JC. 2011. Urban Forest Development in West Africa: Benefits and Challenges. Journal of Biodiversity and Ecological Sciences, 1(1): 77-94.

Dasylva M, Ndour N, Ndiaye O, Sambou B. 2017. Analyse de la flore, de la végétation ligneuse et des fonctions des vallées en zone péri-urbaine post-conflit (Ziguinchor, Sénégal). Int. J. Biol. Chem. Sci., 11(1): $\quad 360-377 . \quad$ DOI: http://dx.doi.org/10.4314/ijbcs.v11i1.28

Ganaba S. 2000. Le neem: un arbre dangereux? Echo de la recherche. Eurêka, 32 :13-16, CNRST, Ouagadougou.

Ganaba S. 2011. La Végétation Ligneuse du Sahel (Burkina Faso). Caractérisation, Utilisations, Tests de Restauration et Gestion. Editions Universitaires Européennes,

Gnoumou A, Thiombiano A, Hahn-Hadjali K, Abadouabou B, Sarr M, Guinko S. 2008. Le Parc Urbain Bangr-Wéoogo: une aire de conservation de la diversité floristique au cour de la ville deOuagadougou, Burkina Faso. Flora et VegetatioSudanoSambesica, 11: 35-48.

Guinko S. 1984. Végétation de la Haute-Volta. Thèse de Doctorat d'État ès-Sciences Naturelles, Bordeaux III, p.394.

Guinko S. 2013. Le Neem, Arbre aux Usages Multiples pour le Développement Durable en Milieu Rural au Burkina Faso. Presses Universitaires de Ouagadougou.

Kadéba A, Sambaré O, Soulama S, Thiombiano A, Schmidt M, Boussim IJ. 2014. Typologie spatiale de la végétation sahélienne en relation avec les indicateurs de dégradation au Burkina Faso. Int. J. Biol. Chem. Sci., 8(3): 1049-1064. DOI: 10.4314/ijbcs.v8i3.19

Korbéogo G. 2016. La culture florale à Ouagadougou (Burkina Faso). Les fleurs comme mar queurs d'identités et de mutations urbaines. Revue Anthropologie et Sociétés, 40(2): 227-248. DOI : https://doi.org/10.7202/1037520

Kyalangalilwa B, Boatwright JS, Daru BH, Maurin O, van der Bank M. 2013. Phylogenetic position and revised classification of Acacia s.l. (Fabaceae: Mimosoideae) in Africa, including new combinations in Vachellia and Senegalia. Botanical Journal of the Linnean Society, 172: 500-523. DOI: http://dx.doi.org/10.1111/boj.12047 
Kyle HC, Kimberly AN. 2013. Introducing urban food forestry: a multifunctional approach to increase food security and provide ecosystem services. Landscape Ecol., 28(9): 1649-1669. DOI: 10.1007/s10980-013-9903-Z

Lebrun J-P, Stork A. 1991,1992, 1995, 1997. Enumération des Plantes à Fleurs d'Afrique Tropicale (Vol 1.2. 3. 4). Conservatoire et Jardin Botanique Genève.

Meineke E, Frank S. 2018. Water availability drives urban tree growth responses to herbivory and warming. Journal of Applied Ecology, 55(4): 1701-1713. DOI: $10.1111 / 1365-2664.13130$

Menozzi M-J. 2007. Mauvaises herbes, qualité de l'eau et entretien des espaces. Natures Sciences Sociétés, 15 (2): 144-153.

Moussa S, Boateng Kyereh B, Kuyah S, Tougiani A, Saadou M. 2019. Composition floristique et structure des forêts urbaines des villes sahéliennes: Cas de Niamey et Maradi, Niger. Rev. RAMRES, 7 (00): 5665.

Nero BF, Kwapong NA, Jatta R, Fatunbi O. 2018. Tree Species Diversity and Socioeconomic Perspectives of the Urban (Food) Forest of Accra, Ghana. Sustainability, 10: $3417 . \quad$ DOI: 10.3390/su10103417

N'Zala D, Miankodila P. 2002. Arbres et espaces verts à Brazzaville (Congo). Bois et Forêts des Tropiques, 272(2): 88-92.

Ouédraogo H. 2005. Les plantes exotiques ligneuses introduites dans la ville de Ouagadougou (Burkina Faso). Mémoire de DEA, Université de Ouagadougou, UFR/SVT, p.67.

Ouédraogo B. 2006. La demande de bois-énergie à Ouagadougou: esquisse d'évaluation de l'impact physique et des échecs des politiques de prix. Développement durable et territoire, Varia (2004-2010). DOI : https://doi.org/10.4000/developpementdurable.4151

Polorigni B, Radji RA, Kokou K. 2015. Politique publique de gestion des espaces verts de la ville de Lomé au Togo. Int. J. Biol. Chem. Sci., 9(4): 1888-1901. DOI : http://dx.doi.org/10.4314/ijbcs.v9i4.14

Population Dataset, 2020. Atlas des populations et pays du monde. Burkina Faso https://www.populationdata.net/wp-content/uploads/populationdata-logo.png

Prince W, Fantom N. 2014. World Development Indicators 2014. World Bank Group: Washington, DC.

Radji AR, Kokou K, Akpagana K. 2010. Étude diagnostique de la flore ornementale du Togo. Int. J. Biol. Chem. Sci., 4(20): 491508.

Sacandé M, Sanou L, Beentje H. 2012. Guide d'Identification des Arbres du Burkina Faso. Royal Botanic Gardens: Kew.

Soma S. 2012. Production de plants, utilisations et perception des espèces locales par les pépiniéristes de la ville de Ouagadougou. Mémoire de DEA, UFR/SVT, Université de Ouagadougou, p. 42. De Souza e Silva JL, de Oliveira MTP, Oliveira W, Borges LA, Cruz-Neto O, Lopesa AV. 2020. High richness of exotic trees in tropical urban green spaces: Reproductive systems, fruiting and associated risks to native species. Urban Forestry \& Urban Greening, 50: 126659 . DOI: https://doi.org/10.1016/j.ufug.2020.1266 59

Thiombiano A, Schmidt M, Dressler S, Ouédraogo A, Hahn K, Zizka G. 2012. Catalogue des plantes vasculaires du Burkina Faso. Boissiera, 65: 1-391.

Traoré MY. 2011. Le «propre » et le « sale». Mode de gestion des déchets ménagers et logiques identitaires à Ouagadougou (Burkina Faso). Thèse de doctorat, Département de sociologie, Université de Poitiers.

Vroh BTA, Tiebre MS, N'Guessan KE. 2014. Diversité végétale urbaine et estimation du stock de carbone: cas de la commune $\mathrm{du}$ Plateau Abidjan, Côte d'Ivoire. Afrique Science, 10(3): 329-340. 